\title{
Ação da Betametasona em Ratas Prenhes: Impacto sobre os Niveis de Corticosterona e Glândulas Adrenais Maternas e Fetais
}

\author{
Effect of Betamethasone on Pregnant Rats: Impact on Corticosterone Level \\ and Maternal and Fetal Adrenal Glands \\ Eduardo de Souza, Sérgio Kobayashi, Manuel de Jesus Simões \\ Luiz Camano, Luiz Kulay Júnior
}

\begin{abstract}
RESUMO
Objetivo: a utilização repetitiva do corticóide antenatal objetivando acelerar a maturidade pulmonar fetal tem sido muito empregada no risco de parto prematuro, o que nos motivou a estudar a dosagem de corticosterona no termo e aspectos morfológicos das glândulas adrenais maternas e fetais de ratas albinas submetidas à ação da betametasona na segunda metade da prenhez, para verificar conseqüências dessa terapêutica.

Métodos: utilizamos 30 ratas prenhes, distribuidas em 3 grupos numericamente iguais. As do Grupo I receberam betametasona nos dias 11, 12, 18 e 19 da prenhez. As do Grupo II receberam água destilada nesses dias (grupo controle), e as do Grupo III não receberam qualquer medicamento, constituindo grupo controle de estresse. Foram todas sacrificadas no $20^{\circ}$ dia de prenhez, quando dosamos a corticosterona no sangue das matrizes e extirpamos as glândulas adrenais maternas e fetais para exame de microscopia óptica.

Resultados: a dosagem de corticosterona plasmática foi significantemente menor no grupo tratado com betametasona $(4,8 \mu \mathrm{g} / \mathrm{dL})$, quando comparada aos grupos controles $(17,7$ e 26, 8 $\mu \mathrm{g} / \mathrm{dL}$ ). À microscopia óptica observou-se intensa vacuolização citoplasmática na zona fasciculada das adrenais maternas e fetais no grupo que utilizou a betametasona, indicando intensa supressão adrenal secundária ao uso do medicamento.

Conclusões: o uso repetitivo e prolongado de corticóides, em ratas prenhes, para acelerar a maturidade pulmonar fetal determina supressão adrenal materna e fetal.
\end{abstract}

PALAVRAS-CHAVE: Betametasona. Corticosterona. Supressão adrenal.

Introdução

A prematuridade é considerada o mais sério problema da Obstetrícia em nossos dias, sendo responsável por elevados índices de morbidade e mortalidade perinatais.

O parto em idade gestacional precoce determina o nascimento de uma criança com variáveis graus de imaturidade dos seus órgãos e sistemas. O desenvolvimento pulmonar incompleto é, sem dúvida, um dos aspectos mais comprometedores do prognóstico neonatal.

Universidade Federal de São Paulo - Escola Paulista de Medicina

Correspondência:

Eduardo de Souza

Estrada das Lágrimas 3355, Ipiranga
Com o objetivo de promover a aceleração da maturidade pulmonar fetal, nas gestantes com risco de prematuridade, tem sido proposta a corticoterapia materna.

A investigação clínica sobre esse uso de esteróides iniciou-se com os trabalhos de Liggins ${ }^{1}$, enquanto estudava aspectos do determinismo da parturição. Posteriormente, Liggins e Howie ${ }^{2}$ realizaram o primeiro estudo randômico, duplo cego, em humanos, no qual comprovaram que o uso materno antenatal da betametasona reduziu significantemente a incidência da sindrome do desconforto respiratório e a mortalidade neonatal.

A partir dessas observações iniciais, diversos trabalhos surgiram na literatura, com o intuito de estudar a ação da corticoterapia materna antenatal na aceleração da maturidade pulmonar fetal, com destaque para os estudos colaborativos, 
multicêntricos e de metanálises ${ }^{3-9}$.

A publicação do N.I.H. ("National Institutes of Health") dos Estados Unidos da América ${ }^{10}$, elaborada como consenso, obteve ampla divulgação; obtida com base nas evidências científicas, reconheceu os beneficios da administração antenatal de corticosteróides a fetos com risco de parto prematuro, com redução da síndrome do desconforto respiratório, da mortalidade neonatal e da hemorragia intraventricular. Definiu a faixa de idade gestacional (24 a 34 semanas) em que o uso do corticóide deve ser considerado, independentemente da disponibilidade de terapia de reposição de surfactante exógeno. O tratamento consiste de duas doses de $12 \mathrm{mg}$ de betametasona por via intramuscular com 24 horas de diferença ou quatro doses de $6 \mathrm{mg}$ de dexametasona por via intramuscular com 12 horas de diferença. Deve ser ministrado, exceto se o parto imediato for esperado. $\mathrm{O}$ efeito da terapia dura sete dias.

Após a divulgação desses trabalhos, o uso da corticoterapia em Obstetrícia, com o objetivo de acelerar a maturação pulmonar fetal, se intensificou. A sua utilização, sobretudo em gestações de alto risco, ocasionou aumento na utilização de ciclos repetitivos do medicamento. Isto promoveu o surgimento de aspectos polêmicos, como a real eficiência da repetição dos ciclos e seus efeitos colaterais $^{11}$.

Alguns trabalhos experimentais foram feitos acerca do uso de corticosteróides, também com o intuito de observar seus efeitos nos pulmões dos conceptos $^{12-14}$. Apesar de comprovarem o beneficio, em outros experimentos o uso da dexametasona em animais, na fase tardia da gestação, não mostrou-se inócuo. Determinou menor ganho de peso da matriz e diminuição no peso dos fetos e de alguns de seus órgãos, como coração, figado, pulmões e cérebro ${ }^{15,16}$.

No campo experimental, também realizamos ensaio biológico estudando a ação da betametasona na segunda metade da prenhez da rata albina ${ }^{17}$. No grupo tratado com betametasona no $11^{\circ}, 12^{\circ}$, $18^{\circ}$ e $19^{\circ}$ dia de prenhez, as matrizes exibiram ganho de peso significativamente menor quando comparadas às dos grupos controles. Detectamos, ainda, redução significante dos pesos individuais dos fetos e das placentas no grupo que recebeu o corticosteróide.

A verdadeira razão dessa interferência no crescimento fetal e de órgãos ainda não está bem definida.

Diante desses dados de literatura, considerando a importância da alternativa da corticoterapia na prematuridade, e principalmente preocupados com o aumento progressivo de gestantes com risco de parto prematuro submetidas a ciclos repetitivos de corticosteróides exógenos, ficamos motivados a continuar os estudos experimentais do uso da betametasona na segunda metade da gestação de ratas albinas, buscando avaliar repercussões funcionais e morfológicas sobre as glândulas adrenais maternas e fetais.

\section{Material e Métodos}

Utilizamos ratas albinas (Rattus norvegicus albinus, Rodentia Mammalia), da linhagem EPM1 Wistar, oriundas do Biotério Central da Universidade Federal de São Paulo - Escola Paulista de Medicina, adultas, virgens, com aproximadamente 90 dias de idade, pesando entre 200 e 250 gramas, com regime de alimentação normal (ração Purina-Labina) e água ad libitum.

Todos os animais foram transportados para o biotério, onde foram mantidos em gaiolas de poliuretano medindo $45 \times 30 \times 15 \mathrm{~cm}$, respectivamente, de comprimento, largura e altura, sendo cinco ratas por gaiola. A temperatura ambiente foi de aproximadamente $22^{\circ} \mathrm{C}$ com iluminação artificial de lâmpadas fluorescentes, modelo luz do dia, de 40 watts, sendo o fotoperiodo de 12 horas claro e 12 horas escuro, considerando o período de luz das 7:00 às 19:00 horas.

As fêmeas foram acasaladas na proporção de duas para cada macho, por um período de 12 horas, durante o fotoperíodo escuro. O início da prenhez foi determinado pela presença de espermatozóides na vagina da rata. Este foi considerado o dia 0 (zero) da prenhez.

Trinta ratas com teste de prenhez positivo foram distribuídas aleatoriamente em três grupos, numericamente iguais, a saber:

Grupo I (G I) - ratas que receberam betametasona, por injeção intramuscular, na dose de $1 \mathrm{mg} / \mathrm{kg}$ de peso corporal em solução aquosa de 0,5 mL, nos dias 11, 12, 18 e 19 da prenhez. Foi considerado grupo experimental.

Grupo II (G II) - ratas que receberam $0,5 \mathrm{~mL}$ de água destilada por injeção intramuscular nos dias $11,12,18$ e 19 da prenhez. Foi considerado grupo controle.

Grupo III (G III) - as ratas deste grupo não receberam qualquer fármaco ou veículo do fármaco. Foi considerado como grupo controle de estresse.

Todas as injeções foram realizadas entre 9 e 10 horas da manhã, e a pesagem dos animais foi avaliada com balança de precisão. Todas as ratas foram mantidas nas gaiolas nas mesmas condições de biotério.

Todos os animais, entre 9 e 10 horas da 
manhã do $20^{\circ}$ dia de prenhez, foram decapitados em guilhotina, e $3 \mathrm{~mL}$ de sangue coletados em tubos de centrífuga contendo citrato trissódico na concentração de $23 \mathrm{mM}$, como anticoagulante. O tempo decorrido entre a retirada do animal da gaiola e a sua decapitação foi sempre inferior a 30 segundos.

Os tubos foram colocados a $2500 \mathrm{rpm}$, em ultracentrifuga refrigerada a $4^{\circ} \mathrm{C}$, durante $15 \mathrm{mi}$ nutos, ao fim dos quais o plasma foi separado e estocado a $-20^{\circ} \mathrm{C}$ para ulterior dosagem da concentração plasmática de corticosterona.

De imediato os animais foram submetidos a laparotomia com incisão ampla longitudinal mediana, expondo os órgãos internos. Em seqüência os cornos uterinos foram abertos para extração dos fetos e das placentas.

Três conceptos de cada rata foram aleatoriamente submetidos a laparotomia, e deles foram retiradas as glândulas adrenais. As glândulas adrenais das matrizes também foram imediatamente extirpadas.

Esses órgãos maternos e dos conceptos foram rapidamente seccionados de modo a reduzilos a pequenos fragmentos, que foram imediatamente mergulhados no líquido de Bouin e enviados para preparo da microscopia óptica. Essas amostras permaneceram durante 24 horas no líquido de Bouin. Em seguida, o material foi desidratado em etanol, em concentrações crescentes, diafanizado em xilol e impregnado em paraplast a $59^{\circ} \mathrm{C}$. No paraplast do terceiro banho, as peças foram incluídas e os blocos cortados em micrótomo ajustado para $5 \mathrm{~mm}$. As lâminas foram então coradas pela hematoxilina-eosina $(\mathrm{HE})$. Empregamos, para este estudo, microscópio com ocular de aumento de 10 vezes e objetivas cujos aumentos variaram entre 4 e 100 vezes.

Os exames histológicos das adrenais maternas e fetais visaram avaliar, em detalhe, o aspecto das camadas glomerular e fasciculada.

A concentração de corticosterona plasmática foi determinada por método fluorimétrico, baseado na fluorescência dos corticosteróides em ácido sulfúrico. Fizemos a separação desse hormônio dos demais elementos plasmáticos por extração com diclorometano, hidróxido de sódio 0,1 N e água destilada. Em seguida, adicionamos uma mistura de etanol e ácido sulfúrico, e sua reação com a corticosterona desenvolveu um composto fluorescente cuja leitura foi feita em espectrofotômetro de fluorescência.

A concentração plasmática de corticosterona foi calculada com o auxílio de uma curva padrão e expressa em microgramas de corticosterona por decilitro de plasma $(\mu \mathrm{g} / \mathrm{dL})$.

\section{Método estatistico}

Para a comparação da dosagem de corticosterona plasmática entre os grupos estudados, foi utilizada a análise de variância por postos de Kruskal-Wallis. Quando mostrou diferença significante, esta análise foi complementada pelo teste de comparações múltiplas. Fixou-se em 0,05 ou $5 \%$ o nivel para a rejeição da hipótese de nulidade, assinalando-se com um asterisco os valores significantes.

\section{Resultados}

A Tabela 1 é referente à dosagem de corticosterona plasmática no termo da prenhez. Observamos que o grupo que recebeu a betametasona antenatal (G I) apresentou níveis plasmáticos de corticosterona menores que os dos grupos controles (G II e G III). As médias dos valores são: 4,8 $\mu \mathrm{g} / \mathrm{dL}$ (G I), 17,7 e $26,8 \mu \mathrm{g} / \mathrm{dL}$ nos grupos G II e GIII, respectivamente.

Tabela 1 - Ratas dos grupos estudados (GI, GII e GIII), segundo a dosagem de corticosterona plasmática $(\mu \mathrm{g} / \mathrm{dL})$ no termo.

\begin{tabular}{cccc}
\hline & \multicolumn{3}{c}{ Corticosterona plasmática $(\mu \mathrm{g} / \mathrm{dL})$} \\
& GI & GII & GIII \\
\hline 4,5 & 11,7 & 11,7 \\
& 4,5 & 9,9 & 35,1 \\
& 3,6 & 14,4 & 26,1 \\
& 4,5 & 6,3 & 22,5 \\
& 3,6 & 7,2 & 55,8 \\
& 3,6 & 9,9 & 27,0 \\
& 5,4 & 36,0 & 24,3 \\
& 6,3 & 14,4 & 26,1 \\
Média & 6,3 & 24,3 & 12,6 \\
Desvio padrão & 4,8 & 43,2 & 27,0 \\
\hline
\end{tabular}

Análise de variância por postos de Kruskal-Wallis $H$ calculado $=20,35^{*} \begin{array}{r}\text { (GI } \times \text { GII } \times \text { GIII) } \\ \text { Teste de Comparaçicos Múltiplas }\end{array}$ $\mathrm{GI}<\mathrm{GII}$ e GIII

$\mathrm{Gl}$ - grupo experimental (injeção de betametasona)

GII - grupo controle (injeção de água destilada)

GIII - grupo controle de estresse (sem medicamentos)

Nas figuras mostramos os achados microscópicos das glândulas adrenais maternas e fetais (microscopia óptica). Verifica-se que as adrenais maternas e fetais do grupo experimental - sub- 
metido à ação da betametasona durante a segunda metade da prenhez - (Figuras $1 \mathrm{~b}$ e $2 \mathrm{~b}$, respectivamente) apresentam-se nitidamente distintas daquelas do grupo controle (Figuras 1 a e $2 a$, res- pectivamente). O citoplasma das células da zona fasciculada sofreu extensa vacuolização, denunciando estado de supressão da adrenal.
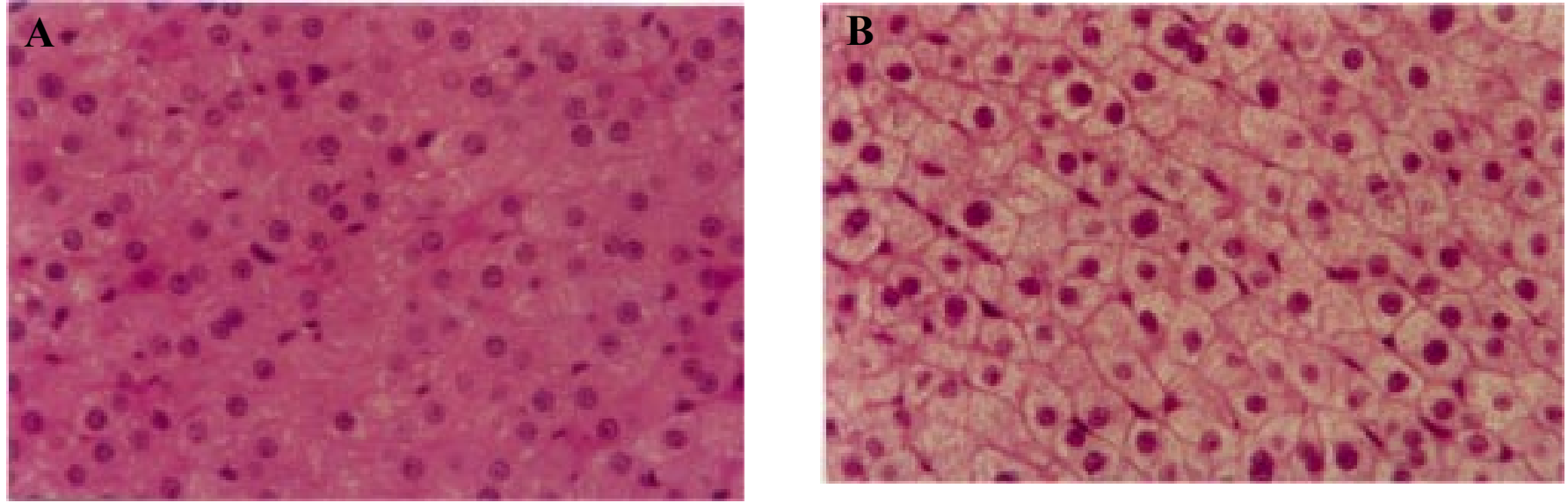

Figura 1(A) - Fotomicrografia de parte da adrenal de rata no $20^{\circ}$ dia de prenhez (grupo controle). Observar o citoplasma das células da zona fasciculada, corado em róseo (HE $550 \mathrm{X}$ ) (B) - Fotomicrografia de parte da adrenal de rata no $20^{\circ}$ dia de prenhez submetida a corticoterapia (grupo experimental). Notar o citoplasma das células da zona fasciculada, vacuolizados (HE 550X).
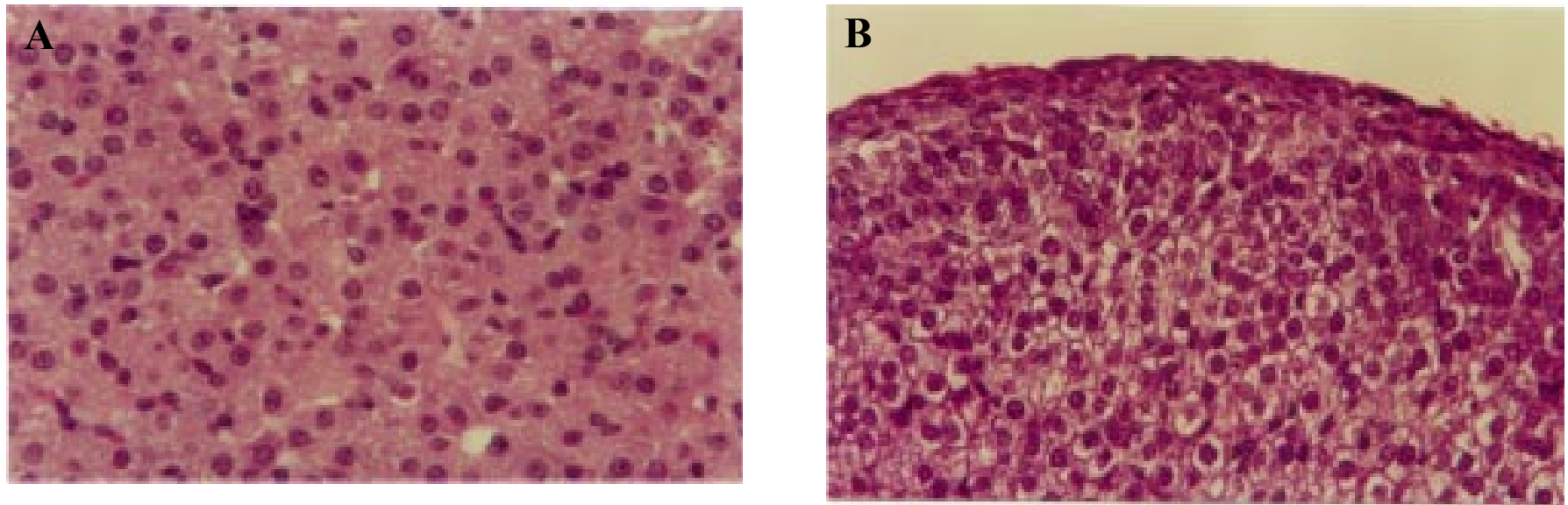

Figura 2(A) - Fotomicrografia de parte da adrenal de feto de rata no $20^{\circ}$ dia de prenhez (grupo controle). Observar o citoplasma das células da zona fasciculada, corado em róseo (HE 550X). (B) - Fotomicrografia de parte da adrenal de feto de rata no $20^{\circ}$ dia de prenhez submetida a corticoterapia (grupo experimental). Notar o citoplasma das células da zona fasciculada, vacuolizados (HE 550X).

\section{Discussão}

Há cerca de trinta anos, a corticoterapia materna tem sido utilizada com o objetivo de promover aceleração da maturidade pulmonar fetal. A sua aplicação tem sido cada vez mais freqüente, principalmente nas gestações de risco para prematuridade e na antecipação eletiva do parto pré-termo, para salvaguardar a vida do feto.

A terapia esteróide antenatal é capaz de modificar múltiplos aspectos da fisiopatologia da sindrome do desconforto respiratório. Talvez a produção de surfactante pelo pneumócito do tipo II seja o principal aspecto desse benefício.

Com o objetivo de tentar reproduzir ao máximo a exposição prolongada e cíclica da gestante e seu feto ao corticóide, como ocorre em gestações de alto risco, nas quais por vezes a betametasona é utilizada semanalmente de 24 a 34 semanas de gestação, utilizamos a medicação experimentalmente durante toda a segunda metade da gestação da rata. A nossa opção de dose (1 $\mathrm{mg} / \mathrm{kg}$ de peso corporal por dia) também buscou 
esse objetivo, pois conseguimos realizar apenas dois ciclos de corticóide na rata, mantendo-se o intervalo de sete dias.

Fizemos adaptações inerentes ao trabalho experimental, mas sempre buscando, com os seus resultados, obter ilações clínicas pertinentes.

Analisamos três grupos de ratas e as respectivas ninhadas. O Grupo I foi submetido à corticoterapia, o II apenas à injeção do veículo (água destilada) e o III constituído com o objetivo de observar se as possiveis modificações apuradas poderiam ser decorrentes exclusivamente do estresse da manipulação e da injeção intramuscular.

Pudemos comprovar que no Grupo I ocorreram mudanças significativas nas mães e nos fetos. Essas alterações não foram detectadas nas ratas dos Grupos II e III. Portanto, ao que tudo indica, foram induzidas pelo uso da medicação (betametasona), e não decorrentes da manipulação e estresse dos animais.

Em nosso trabalho, apuramos que a dosagem plasmática de corticosterona materna nas ratas do grupo submetido à betametasona encontrou-se significativamente menor que a dos demais grupos, constatando-se o estado de supressão funcional das adrenais nesse grupo. Lembramos que a corticosterona é o corticóide mais importante na rata. Destacamos ainda que tivemos a preocupação de realizar a decapitação de forma rápida, com o intuito de não estressar o animal e coletamos imediatamente o sangue para o devido processamento.

Outros autores, em estudos com humanos, apuraram diminuição provisória dos niveis de cortisol materno durante a terapêutica com corticosteróide, com retorno à normalidade em poucos dias ${ }^{2,3,7,18,19}$. Esses estudos, entretanto, foram realizados com ciclos simples de corticoterapia antenatal. A supressão adrenal materna e fetal, decorrente do uso repetitivo e prolongado, ainda é pouco estudada. Não há dados suficientes na literatura reveladores das suas conseqüências, a curto e longo prazo, para a mãe e seu concepto. O nosso trabalho buscou observar os resultados com a repetição dos ciclos, acreditando que essa supressão adrenal seja mais grave e duradoura nessas pacientes.

Em nossa análise, com o intuito de aprimorar o estudo das adrenais maternas e fetais, realizamos microscopia óptica e eletrônica dessas glândulas. As adrenais das ratas mães e dos fetos do Grupo I revelaram-se distintas das dos demais grupos. Apuramos extensa vacuolização do citoplasma das células das camadas glomerulosa e fasciculada do córtex, caracterizando acúmulo de colesterol devido ao estado de supressão adrenal pelo uso do corticóide exógeno. Destacamos que todas as ratas mães desse grupo exibiam essas alterações, detectadas também em todas as glândulas adrenais dos seus conceptos analisados.

Nossos resultados podem ser comparados aos de outro experimento também envolvendo ratas, em que os autores concluíram que mesmo uma única dose de dexametasona $(1,5 \mathrm{mg}$ por $\mathrm{kg}$ de peso), no periodo antenatal crítico de desenvolvimento do eixo hipotálamo-hipófise-adrenal, promoveu mudanças na estrutura e função das adrenais fetais, também parcialmente detectadas durante o período neonatal. Apuraram diminuição significativa no peso das adrenais e no volume das zonas capsular, glomerulosa e reticular dos conceptos. Essas alterações seriam decorrentes da diminuição do número de células, além de áreas de necrose e infiltrações por macrófagos, células gigantes e linfócitos. No $14^{\circ}$ dia de vida, o grau dessas mudanças atróficas no córtex adrenal dos conceptos já era de menor gravidade ${ }^{20}$.

Estamos cientes de que os nossos resultados são frutos de trabalho experimental, não podendo ser extrapolados integralmente para a espécie humana. Nosso objetivo é colaborar nesta linha de pesquisa buscando compreender melhor os riscos da terapêutica corticóide prolongada durante a gestação, realizada com objetivos de acelerar a maturidade pulmonar fetal. Outrossim, reavaliações de doses e reversibilidade das lesões orgânicas e funcionais constituem objetivos de futuras publicações nossas.

Entendemos, ainda, que o tema possui outros aspectos não devidamente avaliados, como o desenvolvimento intra-útero do eixo hipotálamohipófise-adrenal e, principalmente, as alterações no seu funcionamento no periodo perinatal.

Esses dados comprovam a necessidade de estudarmos melhor as reações dos conceptos cujas mães receberam o corticóide de forma repetitiva, durante a segunda metade da gestação, por período prolongado após o nascimento.

Gostariamos de assinalar que não apuramos morte materna ou fetal na realização do nosso trabalho, bem como malformações maiores detectáveis ao exame macroscópico cuidadoso. Trabalhos futuros devem ser realizados com o objetivo de avaliar minuciosamente os órgãos dos animais. Alguns estudos envolvendo a dexametasona (corticóide muito semelhante ao utilizado por nós), ministrada tardiamente na prenhez (geralmente após o $17^{\circ} \mathrm{dia}$ ), em doses comparáveis à do nosso experimento, apuraram que essa exposição prematura a altos níveis de esteróide exógeno tem efeito adverso no desenvolvimento celular, particularmente no sistema nervoso central ${ }^{15,16}$. Esses autores admitem a possibilidade de o corticóide 
exógeno agir como um inibidor do desenvolvimento neural, diminuindo a replicação celular, acelerando a morte celular e dificultando as adaptações comportamentais.

Consideramos importante salientar que o nosso trabalho partiu do princípio que o Departamento de Obstetrícia da Universidade Federal de São Paulo tem incentivado o uso da betametasona como medida de destaque para a obtenção de melhor prognóstico neonatal em partos prematuros. Nossa Escola tem sido árdua defensora dessa terapêutica e a tem utilizado de forma criteriosa mas ampla no atendimento à gestação de alto risco para a prematuridade planejada ou não.

Entendemos que os seus beneficios são evidentes, na prática diária, no trato da gestante em trabalho de parto prematuro submetida à tocólise, nas hipertensas, diabéticas, gemeligestas, naquelas com insuficiência istmo-cervical e rotura prematura de membranas, entre outras.

Esses dados recomendam o uso cauteloso, em casos individualizados, do corticóide antenatal com o objetivo de promover a aceleração da maturidade pulmonar fetal. Continuamos, porém, defensores dessa terapêutica diante do risco de prematuridade. Os ciclos repetitivos devem ser evitados. Nosso posicionamento atual é limitar a, no máximo, dois ciclos de corticoterapia.

Temos, principalmente, nos esforçado no apuro do risco do trabalho de parto pré-termo, como forma de evitar a ministração prolongada da betametasona. Entendemos que pesquisas experimentais e clínicas devam prosseguir, particularmente com o objetivo de apurar se ocorrem efeitos maternos e fetais a curto e/ou a longo prazo quando utilizados ciclos tanto isolados quanto repetitivos de betametasona.

\section{SUMMARY}

Purpose: the repetitive use of antenatal corticosteroid therapy for acceleration of fetal lung maturation has been common in cases at risk of preterm delivery. We studied the corticosterone levels at term and the morphologic aspects in the maternal and fetal adrenal glands submitted to the effect of betamethasone in the second half of rat pregnancy in order to verify its consequences.

Methods: thirty female pregnant rats were divided into three groups of ten animals each. Group I received betamethasone on the 11th, 12th, 18th and 19th day of pregnancy. Group II received distilled water on the same days (control group). Group III did not receive any drug (stress control group). All rats were sacrificed on the 20th day of pregnancy when plasma corticosterone levels of dams were assessed and the maternal and fetal adrenal glands were studied by light microscopy.
Results: plasma corticosterone level of dams was lower in the group treated with betamethasone $(4.8 \mu \mathrm{g} / \mathrm{dL})$ when compared with the control groups (17.7 and $26.8 \mu \mathrm{g} / \mathrm{dL}$ ). The light microscopy study revealed cytoplasmatic vacuolation in the fasciculate zone in the maternal and fetal adrenal glands, which indicates adrenal suppression.

Conclusions: the antenatal repetitive and prolonged use of corticosteroids in pregnant rats for acceleration of lung fetal maturation causes maternal and fetal adrenal suppression.

KEY WORDS: Betamethasone. Corticosterone. Adrenal

\section{Referências}

1. Liggins GC. Premature delivery of foetal lambs infused with glucocorticoids. J Endocrinol 1969; 45:515-23.

2. Liggins GC, Howie RN. A controlled trial of antepartum glucocorticoid treatment for prevention of the respiratory distress syndrome in premature infants. Pediatrics 1972; 50:515-25.

3. Effect of antenatal dexamethasone administration on the prevention of respiratory distress syndrome. Am J Obstet Gynecol 1981; 141:276-87.

4. Bauer CR, Morrison JC, Poole WK, et al. A decreased incidence of necrotizing enterocolitis after prenatal glucocorticoid therapy. Pediatrics 1984; 73:682-8.

5. Ohlsson A. Treatments of preterm premature rupture of the membranes: a meta-analysis. Am J Obstet Gynecol 1989; 160:890-906.

6. Crowley P, Chalmers I, Keirse MJ. The effects of corticosteroid administration before preterm delivery: an overview of the evidence from controlled trials. Br J Obstet Gynaecol 1990; 97:11-25.

7. Crowley PA. Antenatal corticosteroid therapy: a metaanalysis of the randomized trials, 1972 to 1994. Am J Obstet Gynecol 1995; 173:322-35.

8. Wright LL, Horbar JD, Gunkel H, et al. Evidence from multicenter networks on the current use and effectiveness of antenatal corticosteroids in low birth weight infants. Am J Obstet Gynecol 1995; 173:263-9.

9. Crowley P. Prophylactic corticosteroids for preterm birth. Cochrane Database Syst Rev 2001; (4):CD000065.

10.Effect of corticosteroids for fetal maturation on perinatal outcomes. NIH Consens Statement 1994; 12:1-24.

11.Vermillion ST, Soper DE, Newman RB. Neonatal sepsis and death after multiple courses of antenatal betamethasone therapy. Am J Obstet 
Gynecol 2000; 183:810-4.

12.Frank L, Lewis PL, Sosenko IR. Dexamethasone stimulation of fetal rat lung antioxidant enzyme activity in parallel with surfactant stimulation. Pediatrics 1985; 75:569-74.

13.Lohninger AK, Böck P, Salzer H, Sevelda P, Lohninger AF. Antenatal betamethasone-doseeffects on fetal rat lung morphology and surfactant. J Perinat Med 1994; 22:319-28.

14.Suen HC, Bloch KD, Donahoe PK. Antenatal glucocorticoid corrects pulmonary immaturity in experimentally induced congenital diaphragmatic hernia in rats. Pediatr Res 1994; 35:523-9.

15.Carlos RQ, Seidler FJ, Slotkin TA. Fetal dexamethasone exposure alters macromolecular characteristics of rat brain development: a critical period for regionally selective alterations? Teratology 1992; 46:45-59.

16.Slotkin TA, Lappi SE, McCook EC, Tayyeb MI, Eylers JP, Seidler FJ. Glucocorticoids and the development of neuronal function: effects of prenatal dexamethasone exposure on central noradrenergic activity. Biol Neonate 1992;
61:326-36.

17. Souza E, Simões MJ, Camano L, Kulay Júnior L. Efeitos da betametasona sobre os fetos e placentas da rata albina. Rev Bras Ginecol Obstet $2001 ; 23: 93-7$.

18. Whitt GC, Buster JE, Killiam AP, Scragg WH. A comparison of two glucocorticoid regimens for acceleration of fetal lung maturation in premature labor. Am J Obstet Gynecol 1976; 124:479-82.

19.Haning RV Jr, Curet LB, Poole WK, Boehnlein LM, Kuzma DL, Meier SM. Effect of fetal sex and dexamethasone on preterm maternal serum concentrations of human chorionic gonadotrophin, progesterone, estrone, estradiol and estriol. Am J Obstet Gynecol 1989; 161:1549-53.

20.Hristic M, Kalafatic D, Plecas B, Jovanovic V. The effect of dexamethasone on the adrenal gland in fetal and neonatal rats. J Exp Zool 1995; 272:281-90.

\section{COMUNICADO AOS ASSOCIADOS E LEITORES}

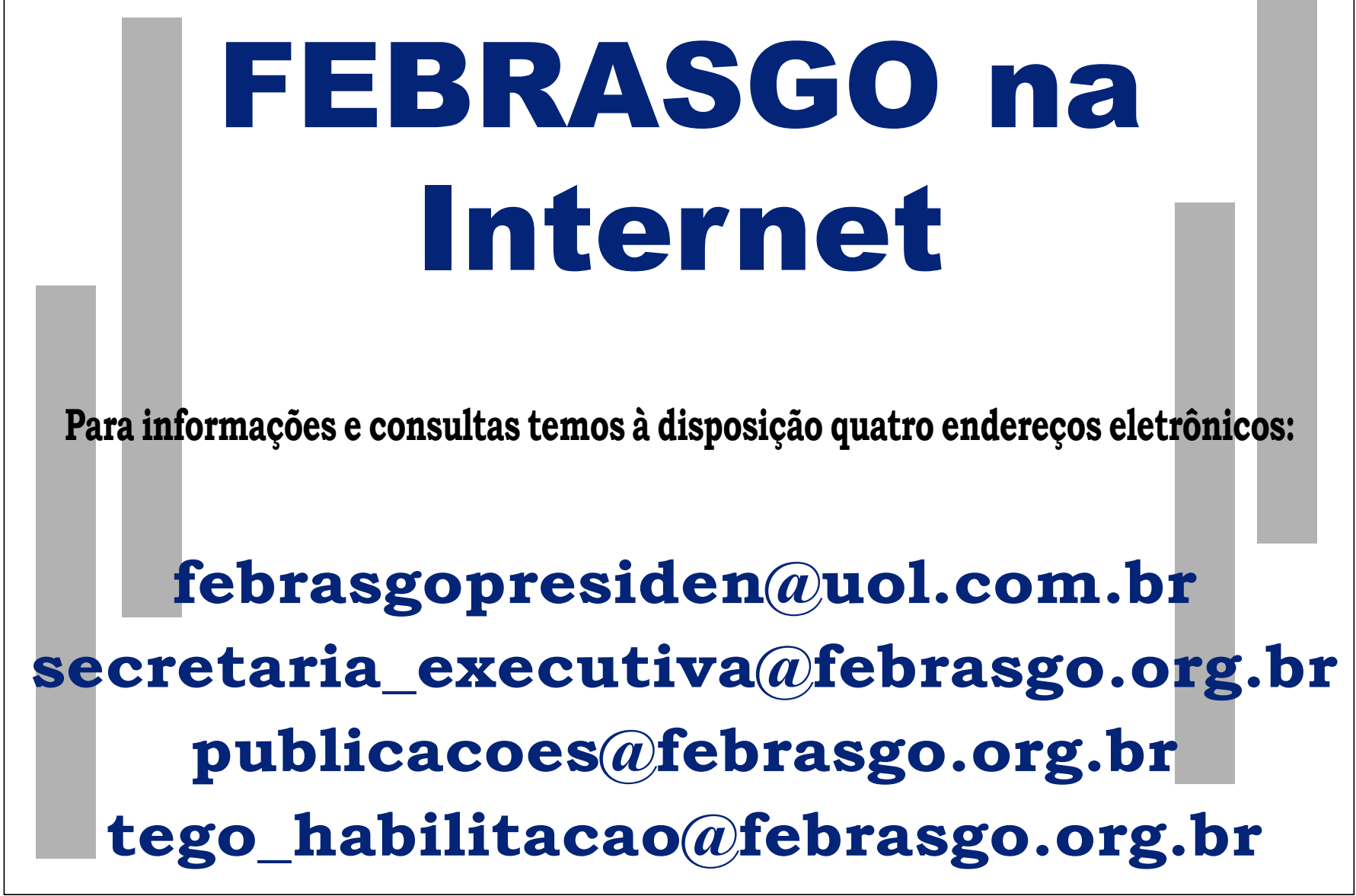

